\title{
OPTIMIZATION OF INDUCED VOLTAGE ON BURIED PIPELINE FROM HV POWER LINES USING GRASSHOPPER ALGORITHM (GOA)
}

\author{
Khadidja BOUALLAG, Rabah DJEKIDEL, Sid Ahmed BESSEDIK
}

\author{
Laboratory for Analysis and Control of Energy Systems and Electrical Systems LACoSERE, Laghouat \\ University (03000), Algeria, k.bouallag@lagh-univ.dz
}

\begin{abstract}
The buried metallic pipeline which parallels to the HV power line is subject to induced voltages from the AC currents flowing in the conductors, these voltages can affect the operating personnel, pipeline associated equipment, and the pipeline integrity. This paper analyses the induced voltage and current on the buried pipeline running parallel to $\mathrm{HV}$ power lines. It also presents an optimization procedure of different parameters that affect the level of the induced voltage in the pipeline during normal operating conditions. A comparison study between the proposed optimization algorithms (GOA, GE, DE and PSO) is done with a maximization of a given objective function. The simulation results establish that the GOA algorithm provides a faster convergence and better solution than the other optimization algorithms. Thus, the statistical analysis according to Friedman's rank test confirmed the superiority of this proposed algorithm. Furthermore, the results show that the parameters optimization of the metallic pipeline is an effective approach to provide the best performance for mitigation which is generally sufficient to reduce the induced voltage experienced by the buried metallic pipeline to enforce the safety limit.
\end{abstract}

Keywords: Buried pipeline, Grasshopper Optimization algorithm (GOA), HV power line, AC induced voltage.

\section{INTRODUCTION}

Installing pipelines in energy utility corridors containing HV power lines subjects the buried metallic pipelines to induced AC voltages. This can be transferred by the electrical interference due to the inductive and conductive effects. The inductive coupling is the most important among those both effects of an $\mathrm{H} \mathrm{V}$ power line on a buried steel pipeline; it is the result of the time-varying electromagnetic induction produced by the HV power line currents. These induced AC voltages can affect the operating personnel, pipeline associated equipment, and pipeline cathodic protection systems [1-7]. In order to minimize the possibility of $\mathrm{AC}$ corrosion and to ensure personnel safety, many standards and regulations provide safety limits relevant to the inductive coupling which can be tolerated on steel buried pipelines [8-11], the European Standard Regulation (CENELEC) sets the limit of the induced voltage on a pipeline to $60 \mathrm{~V}$ under operating conditions [11]. On the other hand, NACE imposes a stricter limit of $15 \mathrm{~V}$ under operating conditions [10].

In the steady-state analysis for a buried metallic pipeline, it has been found that the induced voltage and current on the pipeline are dependent on many parameters, such as resistivity of the soil, relative permeability of the pipeline and, resistivity of pipeline coating, they can also depend on other parameters. This study aims to find the optimized parameters which offer a reduced induced voltage on the buried pipeline, an intelligent algorithm employing Grasshopper Optimization (GOA) is used to get the optimal parameters for use in the analysis method. Grasshopper Optimization Algorithm (GOA) is a novel dominant meta-heuristic algorithm proposed by S. Saremi et al. in 2017 for optimization problems [12]. It mimics grasshopper swarm's behavior and their social interaction, three factors influencing GOA algorithm, wind direction, gravity, and social interaction [13]. In the four recent years, the GOA algorithm is widely applied to various optimization problems in a variety of industrial fields, such as power systems control, renewable energy resources, electrical energy consumption and highvoltage transmission system, due to its easy deployment, high accuracy and effectiveness. It is used to solve the single objective and multi objective optimization problems, and it was tested for constrained and unconstrained test functions. The results show that the GOA algorithm could provide very competitive results, the good robustness and the superior performance of this algorithm was confirmed compared with other optimization algorithms [14-20]. But, at the same time, the GOA algorithm has some shortcomings: (1) original linear convergence parameter causes the processes of exploration and exploitation unbalanced; (2) unstable convergence speed; and (3) easy to fall into the local optimum [21,22].

In this case study, in order to verify the robustness and the effectiveness of the proposed GOA 
algorithm, the obtained result is compared with genetic algorithm (GA), differential evolution (DE) and particle swarm optimization (PSO) algorithms.

\section{INDUCTIVE COUPLING}

The inductive interference between the HV power line and metallic pipeline is the result of the magnetic field generated by the currents flowing within the HV power line conductors; this magnetic coupling is quantified by the mutual impedance of the two circuits and is represented as distributed induced electromotive forces (EMF) sources on the pipeline, this induced electromotive forces cause currents circulation on the metallic pipeline and voltages between the pipeline and the surrounding earth. In inductive coupling, the metallic pipeline acts as the single turn secondary of an air-core transformer in which the HV overhead power line is the primary, as shown in Figure 1 [23-25].

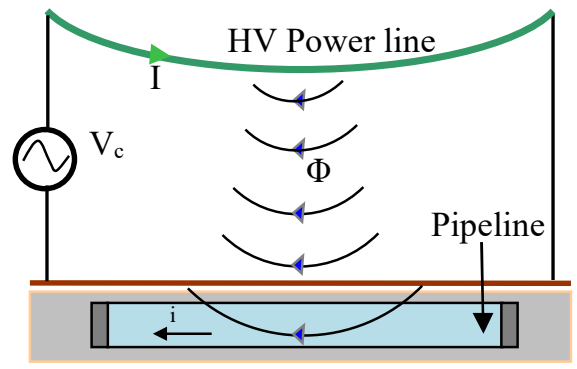

Fig. 1. Inductive coupling between buried pipeline and $\mathrm{HV}$ overhead power line

\section{NODAL NETWORKA NALYSIS}

In this case, the nodal network analysis is used for the induced voltage calculation on the buried pipeline, it based on the $\pi$ type concentrated equivalent circuit impedance matrix. Therefore, this technique offers more robust and reliable results. This induced voltage on the buried pipeline is caused by the induced electromotive force (EMF) produced by the inductive coupling due to the HV overhead power line. Where the buried pipeline is located inside the zone of influence parallel to nearby $\mathrm{HV}$ power line, it is required to model the complex system (buried pipeline, power conductors and soil) and determination of its electrical characteristics, a metallic pipeline can be considered as a long lossy transmission conductor of known geometrical dimensions and physical characteristics as shown in Figure 2 [24, 25]. The equations of the pipeline-ground circuit can be written as follows [8, 24-29]:

$V(x)-z d x . I(x)+E(x) d x-[V(x)+d V(x)]=0$

Dividing (1) by $d x$, therefore,

$$
\frac{d V(x)}{d x}+z \cdot I(x)-E(x)=0
$$

More so, considering the Figure 2 and applying Kirchhoff's current law to node c,

$$
I(x)-d I(x)=y d x \cdot V(x)+I(x)
$$

Dividing through by $d x$,

$$
\frac{d I(x)}{d x}+y V(x)=0
$$

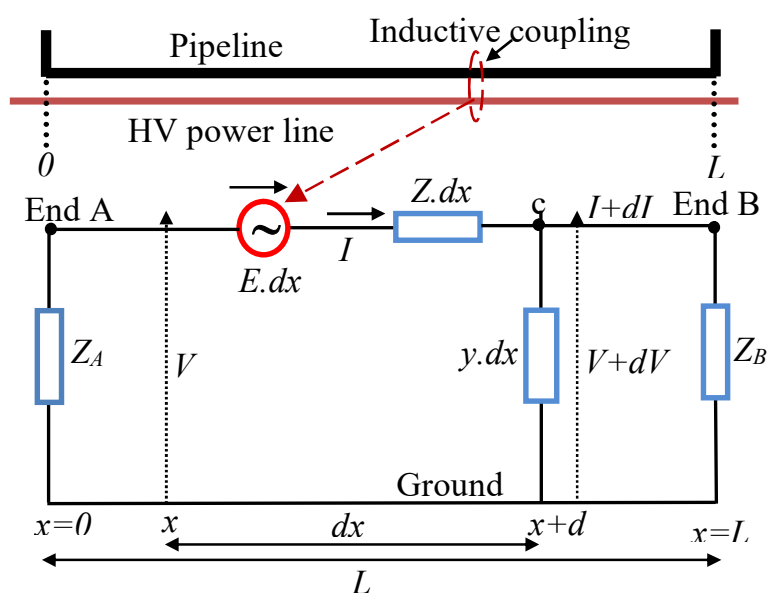

Fig. 2. Representation of the circuit formed by the pipeline and the earth

By differentiating the two complex Equations of power lines (2) and (4) with respect to $\mathrm{x}$ and using the original equations, we obtain two-second order differential equations [8, 24-29]:

$$
\begin{aligned}
& \frac{d^{2} V(x)}{d x^{2}}-y \cdot z \cdot V(x)-\frac{d E(x)}{d x}=0 \\
& \frac{d^{2} I(x)}{d x^{2}}-y \cdot z \cdot I(x)+y \cdot E(x)=0
\end{aligned}
$$

The general solution of equations (5) and (6) is given by the following relations:

$$
\begin{gathered}
V(x)=-Z_{c}\left(A \cdot e^{\gamma x}-B e^{-\gamma x}\right) \\
I(x)=\left(A \cdot e^{\gamma x}+B e^{-\gamma x}\right)+\frac{E}{z_{c}}
\end{gathered}
$$

Where, $\gamma$ is the propagation constant of the buried pipeline; $Z_{c}$ is the characteristic impedance of the buried pipeline, they are given by [8, 24-29]:

$$
\begin{aligned}
& \gamma=\sqrt{z \cdot y} \\
& Z_{c}=\sqrt{\frac{z}{y}}
\end{aligned}
$$

The series impedance per unit length with earth return of the circuit pipeline and earth is given by $[8$, 26]: 


$$
\begin{aligned}
& z=\frac{\sqrt{\rho_{p} \cdot \mu_{0} \cdot \mu_{r} \cdot f}}{3.163 r_{p}}+\pi^{2} \cdot 10^{-4} \cdot f+ \\
& j\left[\frac{\sqrt{\rho_{p} \cdot \mu_{0} \cdot \mu_{r} \cdot f}}{3.163 r_{p}}+4 . \pi^{2} \cdot 10^{-4} \cdot f \log _{e}\left(\frac{D_{e}}{r_{p}}\right)\right]\left[\frac{\Omega}{k m}\right]
\end{aligned}
$$

Where, $r_{p}$ is the pipeline's radius; $\mu_{r}$ is the relative permeability of the pipeline's metal; $\rho_{p}$ is the pipeline's resistivity.

The parallel admittance per unit length of the circuit pipeline and earth is computed using the following formula $[8,26]$ :

$$
y=\frac{\pi \cdot D_{p}}{\rho_{c} \cdot \delta_{c}}+j \cdot \omega \cdot \frac{\varepsilon_{0} \cdot \varepsilon_{r} \cdot \pi \cdot D_{p}}{\delta_{c}}\left[\frac{S}{k m}\right]
$$

The series impedance and the parallel admittance per unit length for buried pipeline can represent in complex form as follows:

$$
\begin{aligned}
& z=|z| \cdot e^{j \cdot \theta_{z}} \\
& y=|y| \cdot e^{j \cdot \theta_{y}}
\end{aligned}
$$

Where, $|z|$ and $|y|$ are the magnitudes of the series impedance and parallel admittance; $\theta_{y}$ and $\theta_{y}$ are the phases of the series impedance and parallel admittance, respectively.

Where, A and B are two constants determined by the boundary conditions (conditions existing at the ends of the pipeline section). They can be expressed as follows [8, 24-29].

$$
\begin{aligned}
& A=\frac{E}{2 . Z} \cdot \frac{\left(1+v_{1}\right) \cdot v_{2}-\left(1+v_{2}\right) e^{\gamma \cdot L}}{e^{-2 \cdot \gamma \cdot L}-v_{1} \cdot v_{2}} \\
& B=\frac{E}{2 . Z} \cdot \frac{\left(1+v_{2}\right) \cdot v_{1}-\left(1+v_{1}\right) e^{\gamma \cdot L}}{e^{-2 \cdot \gamma \cdot L}-v_{1} \cdot v_{2}} \cdot e^{\gamma \cdot L}
\end{aligned}
$$

Where, $v_{1}$ and $v_{2}$ are respectively the coefficients of reflection at the entry and at the exit of the pipeline section, they are given by the following relations [8, 24-29]:

$$
\begin{gathered}
v_{1}=\frac{Z_{A}-Z_{c}}{Z_{A}+Z_{c}} \\
v_{2}=\frac{Z_{B}-Z_{c}}{Z_{B}+Z_{c}}
\end{gathered}
$$

For a pipeline section that continues to run for a few kilometers beyond the parallel routing with the HV overhead power line without earthing [8, 24-29].

For this case,

$$
Z_{A}=Z_{B}=Z_{c}
$$

We obtain;

$$
v_{1}=v_{2}=0
$$

This leads to the following Equations [8, 22-27]:

$$
\begin{aligned}
& V(x)=\frac{E}{2 \cdot \gamma}\left(e^{\gamma(x-L)}-e^{-\gamma x}\right) \\
& I(x)=\frac{E}{2 \cdot Z_{c}}\left(2-e^{\gamma(x-L)}-e^{-\gamma x}\right)
\end{aligned}
$$

More generally, the solutions of this system, the $\mathrm{AC}$ induced voltage and current levels presented on the pipeline (including magnitude and phase angle) can be expressed in complex form as described below,

$$
\begin{aligned}
& V_{(x)} \cdot e^{j \cdot \theta_{V_{(x)}}}=\frac{1}{2}\left(\frac{|E| \cdot\left|\left(e^{\gamma(x-L)}-e^{-\gamma x}\right)\right|}{|\gamma|}\right) \cdot e^{j \cdot\left(\theta_{E}+\theta_{\left(e^{(x-L)}-e^{-\gamma x}\right)}-\theta_{\gamma}\right)} \\
& I_{(x)} \cdot e^{j \cdot \theta_{I_{(x)}}}=\frac{1}{2}\left(\frac{|E| \cdot\left|2-e^{\gamma(x-L)}-e^{-\gamma x}\right|}{\left|Z_{c}\right|}\right) \cdot e^{j \cdot\left(\theta_{E}+\theta_{\left(2-e^{-(x-L L)}-e_{e}^{-\gamma x}\right)}-\theta_{Z_{c}}\right)}
\end{aligned}
$$

The maximum induced voltage on the buried pipeline occurs at the ends of the parallel routing at $\mathrm{x}$ $=\mathrm{L}$ and $\mathrm{x}=0$, and is given by [8, 24-29]:

$$
\left|V_{(0)}\right|=\left|V_{(L)}\right|=U_{\max }=\frac{E}{2 \cdot \gamma}\left(1-e^{-\gamma L}\right)
$$

Outside the exposure, the pipeline voltage and current decrease according to the following exponential function [28]:

$$
\begin{aligned}
& V(x)=U_{\max } \cdot e^{-\gamma x} \\
& I(x)=\frac{U_{\max } \cdot \gamma}{Z_{c}} \cdot e^{-\gamma x}
\end{aligned}
$$

with: $\mathrm{x}$ is the co-ordinate outside the parallel section.

The total longitudinal electromotive force induced on the buried pipeline due to the three-phase currents and the two earth wires currents is given by $[8,27,29]$ :

$$
E=-I_{a} \cdot Z_{p a}-I_{b} \cdot Z_{p b}-I_{c} \cdot Z_{p c}-I_{g_{1}} \cdot Z_{p g_{1}}-I_{g_{2}} \cdot Z_{p g_{2}}
$$

Where, the indices 'a', 'b','c', 'g1', 'g2' , and ' $p$ ' represent the power lines' phase conductors, power lines' earth wires, and pipeline, respectively. Since the earth wires have zero voltage drops, the voltages are given by the equation below:

$$
\begin{aligned}
& \Delta V_{g 1}=I_{a} \cdot Z_{g 1 a}+I_{b} \cdot Z_{g 1 b}+I_{c} \cdot Z_{g 1 c}+I_{g 1} \cdot Z_{g_{1} g_{1}}+I_{g 2} \cdot Z_{g_{1} g_{2}} \approx 0 \\
& \Delta V_{g 2}=I_{a} \cdot Z_{g 2 a}+I_{b} \cdot Z_{g 2 b}+I_{c} \cdot Z_{g 2 c}+I_{g 1} \cdot Z_{g_{2} g_{1}}+I_{g 2} \cdot Z_{g_{2} g_{2}} \approx 0
\end{aligned}
$$

Therefore, we can deduce from Equation (29) the currents in the earth wires; we can write them as follows:

$$
\begin{aligned}
& I_{g_{1}}=-I_{a} \cdot \frac{Z_{g 1 a}}{Z_{g_{1} g_{1}}+Z_{g_{1} g_{2}}}-I_{b} \cdot \frac{Z_{g 1 b}}{Z_{g_{1} g_{1}}+Z_{g_{1} g_{2}}}-I_{c} \cdot \frac{Z_{g 1 c}}{Z_{g_{1} g_{1}}+Z_{g_{1} g_{2}}} \\
& I_{g_{2}}=-I_{a} \cdot \frac{Z_{g 2 a}}{Z_{g_{2} g_{1}}+Z_{g_{2} g_{2}}}-I_{b} \cdot \frac{Z_{g 2 b}}{Z_{g_{2} g_{1}}+Z_{g_{2} g_{2}}}-I_{c} \cdot \frac{Z_{g 2 c}}{Z_{g_{2} g_{1}}+Z_{g_{2} g_{2}}}(30)
\end{aligned}
$$


Thus, substituting theses values into the Equation (28) and combining terms, we obtain the equation of the induced electromotive force, which is presented as:

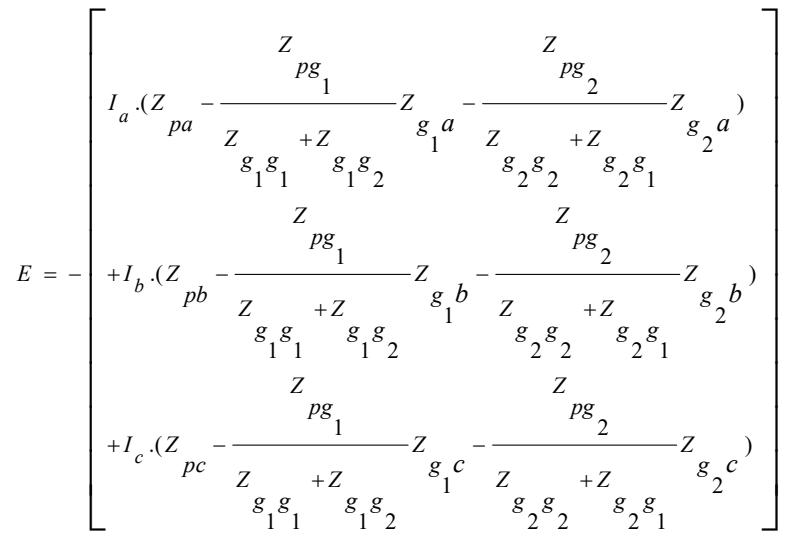

In low frequencies, the self and mutual impedances with earth return of the conductors are obtained according to Carson-Clem's formulae [29]:

$$
\begin{aligned}
& Z_{i i}=R_{i}+\pi^{2} \cdot f \cdot 10^{-4}+j \cdot \omega \cdot 2 \cdot 10^{-4}\left[\ln \left(\frac{D_{e}}{R_{G M}}\right)\right]\left[\frac{\Omega}{k m}\right] \\
& Z_{i j}=\pi^{2} \cdot f \cdot 10^{-4}+j \cdot \omega \cdot 2 \cdot 10^{-4}\left[\ln \left(\frac{D_{e}}{d_{i j}}\right)\right]\left[\frac{\Omega}{k m}\right]
\end{aligned}
$$

Where, $R_{i}$ is the DC resistance per unit length of conductor in $(\Omega / \mathrm{km}), R_{G M}$ is the geometric mean radius of the conductor in $(\mathrm{m}) ; d_{i j}$ is the distance between the conductor $i$ and the conductor $j ; D_{e}$ is the complex penetration depth and is given by [29]:

$$
D_{e}=\sqrt{2} \cdot \delta \cdot e^{-j \pi / 4}, \delta=503 \sqrt{\frac{\rho_{s}}{f}}
$$

Where, $\delta$ is the skin depth of the ground; $\rho_{S}$ is the earth resistivity (assumed uniform) $(\Omega . \mathrm{m}) ; f$ is the frequency of the current in $(\mathrm{Hz}) ; j$ is the imaginary number.

\section{GRASSHOPPER OPTIMIZATION ALGORITHM (GOA)}

The grasshopper optimization algorithm (GOA) is a novel meta-heuristic algorithm for global optimisation inspired by different movement characteristics in larval and adult phases of grasshoppers, in the larval stage, the grasshopper progresses in slack, short paces where in the adulthood stage, the grasshopper progresses quickly with long paces. The GOA algorithm simulates the swarming behaviour of grasshoppers and based on three factors, the social interaction, gravity force and wind advection to control the movement of the grasshopper position, the target of food is also an important influence factor. With the influence of the three concepts, the migration process of the grasshopper swarm is divided into two stages which are exploration and exploitation. In GOA algorithm, the position of the grasshoppers in the swarm represents a possible solution of a given optimization problem [12, 31-34].

The mathematical model employed to simulate the swarming behaviour of grasshoppers is given as follows:

$$
X_{i}=S_{i}+G_{i}+A_{i}
$$

Where, $X_{i}$ is the position of the $i^{\text {th }}$ grasshopper, $S_{i}$, $G_{i}$, and $A_{i}$ indicate the social interaction, gravity force, and wind advection on the $i^{\text {th }}$ grasshopper, respectively. Note that to prepare random behavior, the equation written as:

$$
X_{i}=r 1 . S_{i}+r 2 . G_{i}+r 3 . A_{i}
$$

Where, $r_{1}, r_{2}$ and $r_{3}$ are random numbers that interval rang $[0,1]$.

The social interaction force between each grasshopper and the other grasshopper can be defined as following [12, 31-35].

$$
S_{i}=\sum_{j=1}^{N} s\left(d_{i j}\right) \vec{d}_{i j} \quad j \neq i
$$

Where, $d_{i j}$ is the distance between the grasshopper $i^{\text {th }}$ and grasshopper $\mathrm{j}^{\text {th }}$, calculated as,

$$
d_{i j}=\left|x_{j}-x_{i}\right|
$$

$\vec{d}_{i j}$ is a unit vector from the $\mathrm{i}^{\text {th }}$ grasshopper to the $\mathrm{j}^{\text {th }}$ grasshopper which can be defined as,

$$
\vec{d}_{i j}=\frac{x_{j}-x_{i}}{d_{i j}}
$$

$N$ is the total number of grasshoppers, and $s$ is a function to define the strength of social forces which can be defined as following [12, 31-34].

$$
s(r)=f e^{\frac{-r}{l}}-e^{-r}
$$

Where, $f$ and $l$ are the intensity of the attraction and the attractive length scale.

The factor of gravity $G_{i}$ is denoted as follows [12, 31-34]

$$
G_{i}=-g \vec{e}_{g}
$$

Where, $g$ is the gravitational constant and $\vec{e}_{g}$ is the unity vector to earth center.

The factor of wind direction $A_{i}$ can be calculated as following [12, 31-34]:

$$
A_{i}=u \vec{e}_{\omega}
$$

Where, $u$ is a constant drift and $\vec{e}_{\omega}$ is a wind direction unity vector.

The position of the grasshopper (Equation 36) can be rewritten as follow:

$$
X_{i}=\sum_{j=1}^{N} s\left(\left|x_{j}-x_{i}\right|\right) \frac{x_{j}-x_{i}}{d_{i j}}-g \vec{e}_{g}+u \vec{e}_{\omega}
$$


In this mathematical model the grasshopper reach the comfort zone very quickly and swarm does not converge to a specified point. To solve the optimization problems, the model presented above should be equipped with special parameters to show exploration and exploitation in different stages of optimization. A modified version of this equation is proposed as follows:

$$
X_{i}=c \sum_{j=1}^{N} c\left(\frac{u b_{d}-l b_{d}}{2} s\left(\left|x_{j}^{d}-x_{i}^{d}\right|\right) \frac{x_{j}-x_{i}}{d_{i j}}+\bar{T}_{d}\right.
$$

Where, $u b_{d}$ and $l b_{d}$ represent the upper and lower boundaries of the search space in the $\mathrm{d}^{\text {th }}$ dimension respectively, and $\bar{T}_{d}$ is the food target position which represents the best fitness position in the $\mathrm{d}^{\text {th }}$ dimension.

The parameter $c$ is called a decreasing coefficient, it is responsible for reducing the comfort zone, repulsion zone, and attraction zone, and it can be calculated as follow [12, 31-34].

$$
c=c_{\max }-l \frac{c_{\text {max }}-c_{\text {min }}}{L}
$$

Where, $c_{\max }$ and $c_{\min }$ are the maximum value and the minimum value of $\mathrm{c}$ respectively; $l$ is the current iteration and $L$ is the maximum number of iterations.

The pseudo-code of the grasshopper algorithm is described as follows [34-36]:

Randomly initialize positions of all (grasshoppers) solutions.

Set the parameters values of the GOA algorithm. Do:

- Evaluate the objective function value (fitness value).

- Determine the best grasshopper among all the grasshopper in the population found so far.

- Update grasshopper's positions according to Equation (44).

- Update the decreasing coefficient according to Equation (45).

- While a satisfactory solution has been found.

Through employing the GOA optimization technique with other algorithms, including GA, DE and PSO to identify the more efficient algorithm (the detailed basic principles of these last three algorithms can be found in references [38, 39]), this one is employed to find the optimal values of the optimization parameters to be inserted in the proposed method to estimate the induced voltage on the buried metallic pipeline by maximizing the objective function mentioned below in Equation (46) [29].

$$
O F=-\sqrt{\left(U_{\max }-U_{o p t}\right)^{2}}
$$

Where, $U_{\max }$ is the initial maximum induced voltage on buried pipeline (before optimization) and $U_{o p t}$ is the new maximum induced voltage after optimization. The negative sign shows the maximization of this objective function [29].

The steps to represent the search GOA optimization algorithm are summarized as follows [39-41]:

Step 1: Set the parameters of the proposed method (data), set initial parameters of GOA algorithm and grasshoppers swarm (population $\operatorname{size}(N),\left(c_{\max }\right), \quad\left(c_{\min }\right)$ maximum number of iteration $(L), f \quad$ (intensity of attraction), $l$ (attractive length scale), the domain search for the optimisation parameters $u b, l b$ ).

Step 2: The optimization process is started by creating a set of random population (solutions $(X))$.

Step 3: Compute the objective function value (fitness function) of these solutions using Equation (46)

Step 4: Update the new current search agent position by Equation (44), the value of the objective function of the new position is calculated.

Step 5: At each iteration $(m)$, the best solution $\bar{T}_{d}$ obtained is assigned according to its value and the coefficient parameter $(c)$ is updating using Equation (45).

Step 6: If there is a better solution, update the best objective function $\bar{T}_{d}$ and corresponding position. Then, determine if the stop criterion is reached $(m \geq L)$. If yes, the iteration is terminated. Else, let $(m=m+1)$, and the iteration will be continued iteratively until the satisfaction of an end criterion.

Step 7: Finally, the position and objective function value of the best globe solution (minimum value in the objective function) is returned when the algorithm reaches to its maximum number of iteration.

\section{STATISTICAL ANALYSIS}

The Friedman statistical test is a nonparametric rank-based method; it is employed to assess the statistical differences between the results obtained by a number of algorithms. In this study, the Friedman test is used to analyze the objective function values obtained using the different algorithms. Also, the Friedman statistical test is performed to see if there is a significant difference among the results obtained by the tested algorithms concerned by the comparison; where if the Asymp. Sig (p-value) associated with the Friedman test is small than the critical value $(P \prec 0.05)$. Hence there is significant difference between the tested algorithms. In addition, the Friedman test compares the average ranks of all algorithms. All tests have completed under the same conditions and parameters values. Generally, for the training/testing methodology, the data set is divided into training set $(80 \%)$ and testing set $(20 \%)[14,39$, 42-46]. 


\section{CASE STUDY}

Consider the single-circuit $275 \mathrm{kV}$ overhead transmission line and a parallel metallic buried pipeline section in the immediate vicinity at a separation distance of $30 \mathrm{~m}$. The pipeline continues for several kilometers after the two ends (A and $\mathrm{B}$ ) of the parallel routing with the $\mathrm{HV}$ overhead power line $(\mathrm{L}=10 \mathrm{Km})$. Beyond the parallel route, the pipeline extends for $5 \mathrm{~km}$ without earthing as shown in Figure 3.

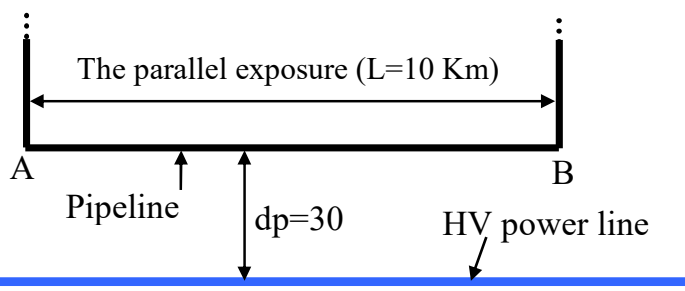

Fig. 3. Representation of the circuit formed by the pipeline and the HV power line

The simplified schematic diagram of the HV overhead transmission line structure used in this proposed study, with the arrangement and geometric details is shown in Figure 4. The threephase currents on the power line have been assumed under balanced operation with the magnitude of $500 \mathrm{~A}$, at nominal frequency $\mathrm{f}=50$ $\mathrm{Hz}$.

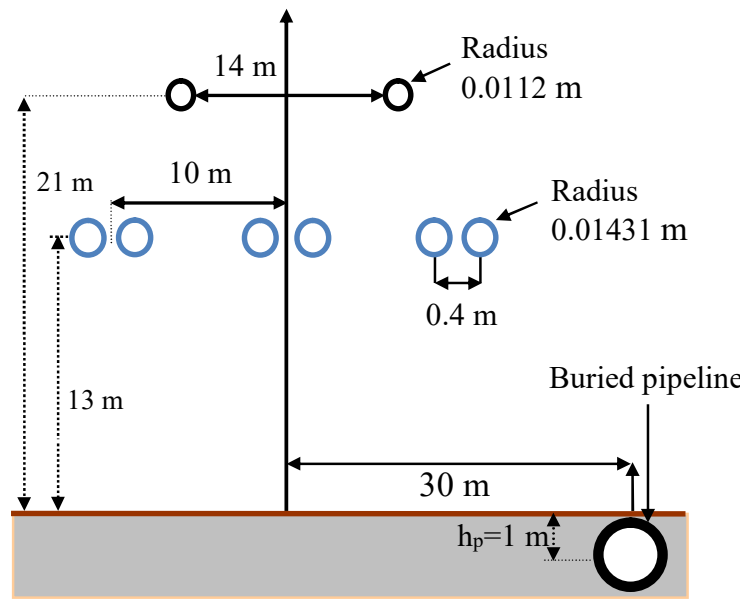

Fig. 4. $275 \mathrm{kV}$ single circuit three phase overhead transmission line

The physical parameters of the buried metallic pipeline used in this analysis are described in Table- 1.

\section{RESULTS AND DISCUSSION}

The first step in this interference analysis should be the calculation of the induced voltage and current levels on the buried metallic pipeline in the steady state operating conditions with no optimization applied.
Tab. 1: Physical parameters of the buried pipeline

\begin{tabular}{|l|l|}
\hline Parameters & Value \\
\hline Resistivity of the soil (ohm.m) $\left(\rho_{s}\right)$ & 100 \\
\hline Relative permeability of the pipeline $\left(\mu_{r}\right)$ & 300 \\
\hline Resistivity of pipeline coating (ohm.m) $\left(\rho_{c}\right)$ & $0.25 \times 10^{7}$ \\
\hline Resistivity of pipeline ( ohm.m) $\left(\rho_{p}\right)$ & $1.7 \times 10^{-7}$ \\
\hline $\begin{array}{l}\text { Relative permittivity of the pipeline coating } \\
\left(\varepsilon_{r}\right)\end{array}$ & 5 \\
\hline Thickness of the coating (m) $\left(\delta_{c}\right)$ & 0.004 \\
\hline Pipeline radius (m) $\left(r_{p}\right)$ & 0.3 \\
\hline
\end{tabular}

Figure 5 shows the profile of AC induced voltage on the buried pipeline, as can be seen in this figure, the induced voltage is negligible at the mid-point of the influence zone and is maximum at the two terminals of the buried pipeline, in the sections of the pipeline that are perpendicular to the HV power line after moving away from the common corridor, it can be observed an exponential decrease in the induced voltage.

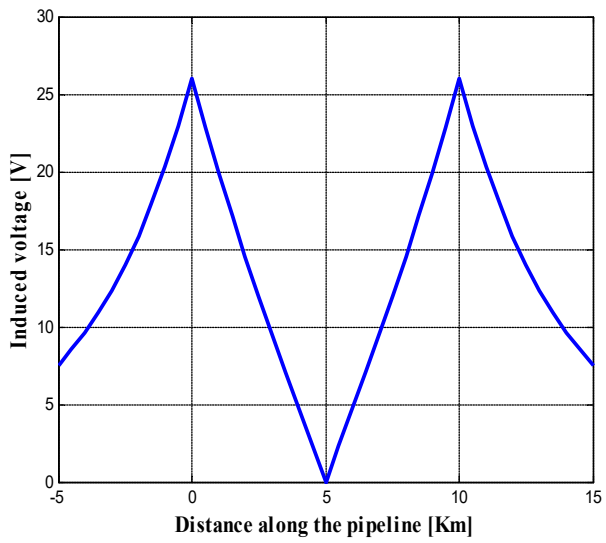

Fig. 5. Induced voltage along the buried pipeline

The profile of the induced current flowing in the buried metallic pipeline is shown in Figure 6. It can be observed that it is considerably reduced at the two terminals of the metallic pipeline, and becomes significant at the mid-point of the zone of influence, beyond the two terminals of the pipeline, at the metallic pipeline sections that run perpendicular to the HV power line, this current decrease gradually.

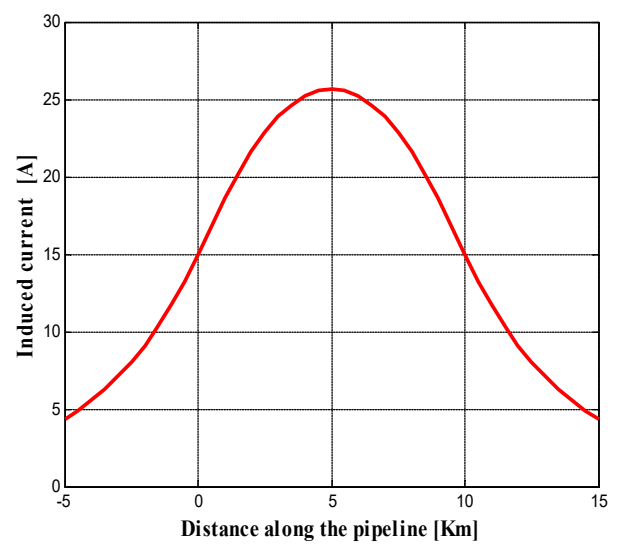

Fig. 6. Induced current along the buried pipeline 
In the second step, a comparison of the performance of the selected optimization techniques (GOA, GA, PSO, DE) is performed in order to obtain the best possible result for the estimated target values of the different parameters that affects the induced voltage on the underground metallic pipeline. For solving this constrained optimisation problem, all the optimisation algorithms were operated using a population size of 20 and 100 iterations. Each one of these parameters defines the search space, considering the upper and lower boundaries. The evolution of these algorithm techniques in the limited search space is guided by an objective function described in equation (46) that is defined to be maximized. These algorithms were coded in Matlab environment (R2012b) on windows 8 platform with I3-4005U Processor 1.7 GHZ processor speed and 4 GB RAM. To ensure a fair comparison, the experiments for each algorithm were repeated 10 times.

The parameter settings of all optimization algorithms are presented in Table- 2 .

Tab. 2: Parameter settings of GOA, GA, PSO and DE algorithms

\begin{tabular}{|c|l|}
\hline Algorithms & Parameters setting \\
\hline GOA & $\begin{array}{l}\text { Search agents }=20 \\
\text { cmax }=1 ; \mathrm{cmin}=0.00001 .\end{array}$ \\
\hline GA & $\begin{array}{l}\text { Population size } \mathrm{N}=20, \\
\text { Mutation probability }=0.2, \\
\text { Crossover probability }=0.4, \\
\text { Number of bits }=25 .\end{array}$ \\
\hline PSO & $\begin{array}{l}\text { Swarm size }=20 ; \mathrm{c} 1=\mathrm{c} 2=2 . \\
\text { Linearly decreases from } 0.6 \text { to } 0.3\end{array}$ \\
\hline DE & $\begin{array}{l}\text { Population size } \mathrm{N}=20 ; \text { step size }=1.5 \\
\text { and crossover probability }=0.95 .\end{array}$ \\
\hline
\end{tabular}

The variation of the objective function that is used to evaluate the optimal parameter values with the number of iterations of the proposed algorithms is illustrated in Figure (7). The optimization process undertaken by these algorithms is increased in order to determine the optimal values of the system parameters (variables) according to the search area, which give a smallest value of the induced voltage on the metallic pipeline. It is evident from this Figure that the objective function value obtained by the GOA algorithm is the highest (maximization), which indicates that the GOA algorithm shows a very fast convergence speed and attains a better optimal solution than GA, PSO and DE algorithms. Therefore, this results comparison confirms that GOA algorithm is more effective for solving this maximization problem of an objective function evaluation. Regarding the computational time for each algorithm, the GA algorithm was the fastest algorithm with 1.306313 seconds; the GOA optimizer algorithm is ranked in the last place with 9.559436 seconds.
In a various previous studies, the results demonstrate that the GOA algorithm has fast convergence speed and highly accurate solutions compared to other algorithms, which indicates the good searching and the performance superiority of GOA algorithm.

The simulation results for the different best parameter values obtained by the GOA optimization algorithm are presented in Figures (8-14), where it becomes obvious that the search of this GOA algorithm converges quickly to the optimum values (optimal solution) of the proposed set of parameters of the buried pipeline.

The best objective function values obtained by the proposed GOA, GA, DE and PSO algorithms and the required computational time for each algorithm to reach the maximum number of iterations are shown in Table-3.

Tab. 3: The optimum values of the GOA, GA, DE and PSO evolutionary algorithms

\begin{tabular}{|l|l|l|}
\hline Parameters & \multicolumn{1}{|c|}{$\begin{array}{c}\text { Initial } \\
\text { value }\end{array}$} & $\begin{array}{l}\text { Optimized } \\
\text { value }\end{array}$ \\
\hline $\begin{array}{l}\text { Resistivity of the soil (ohm.m) } \\
\left(\rho_{s}\right)\end{array}$ & 100 & 500 \\
\hline $\begin{array}{l}\text { Relative permeability of the } \\
\text { pipeline }\left(\mu_{r}\right)\end{array}$ & 300 & 500 \\
\hline $\begin{array}{l}\text { Resistivity of pipeline coating } \\
\left(\text { ohm.m) }\left(\rho_{c}\right)\right.\end{array}$ & $0.25 \times 10^{7}$ & $1 \times 10^{6}$ \\
\hline $\begin{array}{l}\text { Resistivity of pipeline }(\text { ohm.m) } \\
\left(\rho_{p}\right)\end{array}$ & $1.7 \times 10^{-7}$ & $1.4 \times 10^{-6}$ \\
\hline $\begin{array}{l}\text { Relative permittivity of the } \\
\text { pipeline coating }\left(\varepsilon_{r}\right)\end{array}$ & 5 & 13 \\
\hline $\begin{array}{l}\text { Thickness of the coating }(\mathrm{m}) \\
\left(\delta_{c}\right)\end{array}$ & 0.004 & 0.0028 \\
\hline Pipeline radius $(\mathrm{m})\left(r_{p}\right)$ & 0.3 & 0.45 \\
\hline
\end{tabular}

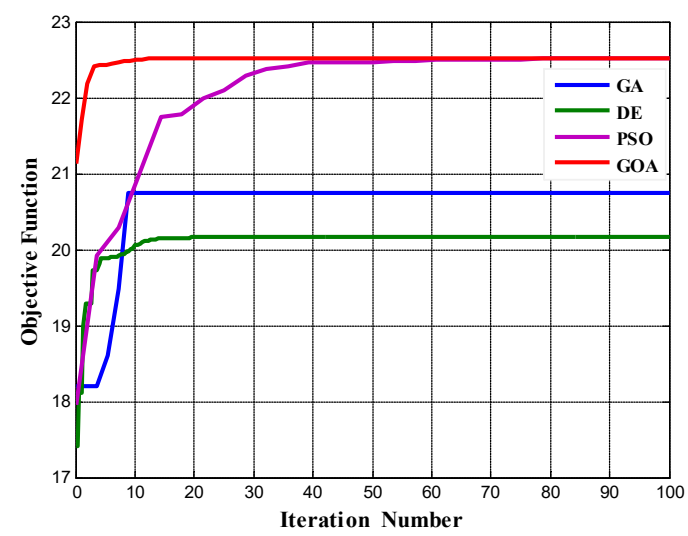

Fig. 7. Objective function variation with number of iterations

The final simulation results of the optimization procedure for the pipeline parameters are summarized in the Table-4 below, 
Tab. 4: Comparison between the original and optimized values

\begin{tabular}{|c|l|l|}
\hline Algorithms & Best solution & Computational time (s) \\
\hline GOA & 17.65 & 9.559436 \\
\hline GA & 15.1 & 1.306313 \\
\hline DE & 15.08 & 3.971845 \\
\hline PSO & 15.8 & 4.038005 \\
\hline
\end{tabular}

The latest step, it is observed from the results presented in Table-4 that the performance of the proposed GOA algorithm is better than the other competitive algorithms. However, it is necessary to conduct the statistical tests like Friedman rank test to prove the significance of the proposed GOA algorithm.

The statistical analysis of results procured using GA, DE, PSO and GOA algorithms are exhibited in Table-5.

Tab. 5: Statistics and Mean ranking coefficient of the Friedman's test for each algorithm.

\begin{tabular}{|l|c|l|l|}
\hline \multicolumn{2}{|c|}{ Test statistics } & Algorithms & $\begin{array}{l}\text { Mean } \\
\text { rank }\end{array}$ \\
\hline $\mathrm{N}$ & 7 & GA & 8 \\
\hline $\begin{array}{l}\text { Friedman Chi-squared } \\
\text { statistic }\end{array}$ & 75.8759 & DE & 11 \\
\cline { 1 - 3 } Degrees of freedom df & 3 & PSO & 5 \\
\hline Asymp. sig. (P value) & $2.3516 \mathrm{e}-16$ & $\begin{array}{l}\text { Proposed GOA } \\
\text { Algorithm }\end{array}$ & 2 \\
\hline Sigma & 3.5291 & . & \\
\hline
\end{tabular}

As seen from Table-5 and after analyzing the results of the Friedman test, the GOA algorithm attains the lowest rank, which means the better performance of this algorithm. Thus, it may be concluded that GOA algorithm provide better quality solution when compared to other optimization techniques.

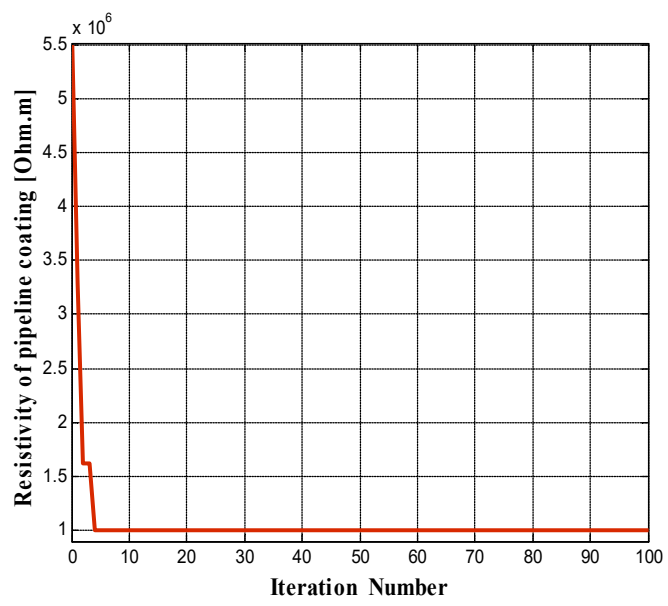

Fig. 10. Convergence to optimal value of the pipeline coating resistivity

To maintain the induced voltage on the buried pipeline within a permissible limit, as observed from Table 4, the optimized value of the relative permeability of the pipeline steel increases compared to the initial value.

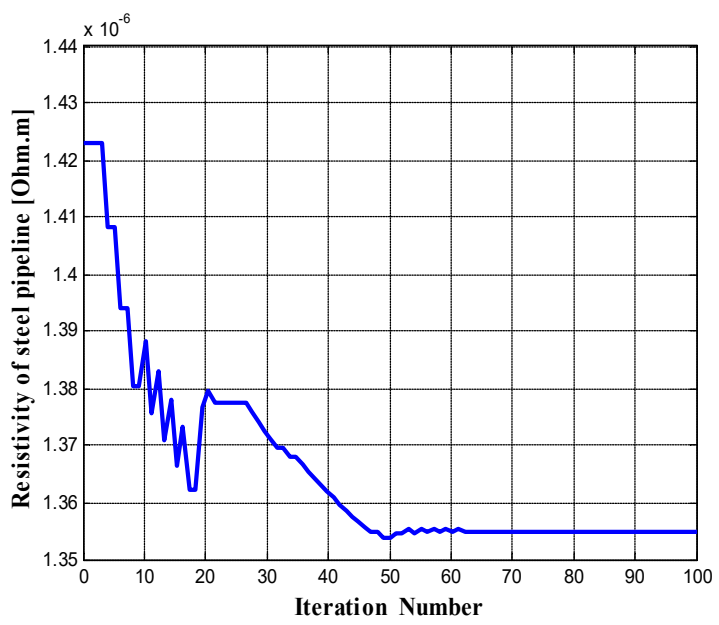

Fig. 11. Convergence to optimal value of the resistivity of steel pipeline

In order to reduce the induced voltage on the pipeline to an acceptable level, it can be seen from Table 4 that the optimized value of the pipeline coating resistivity tends to decrease in comparison with the original value.

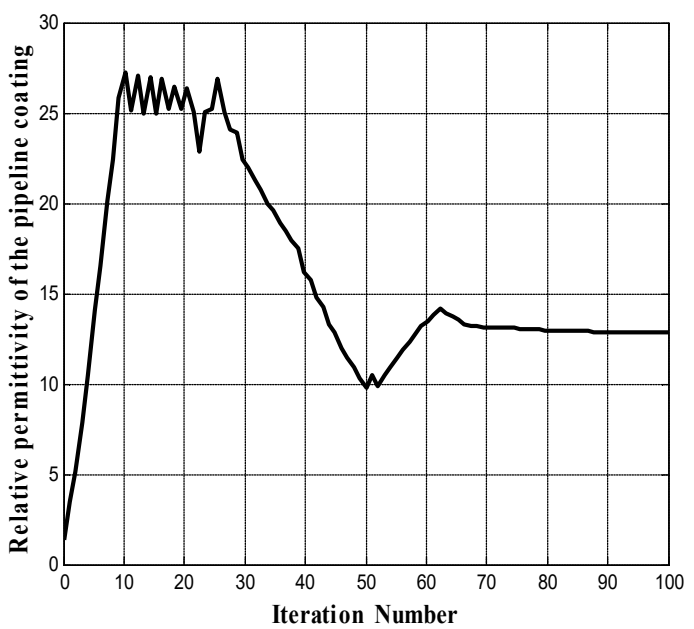

Fig. 12. Convergence to optimal value of the relative permittivity of the pipeline coating

In order to reduce the magnitude of the induced voltage on the buried pipeline to a permissible limit, as presented in Table 4, the optimized value of the resistivity of the steel pipeline just increased compared to the original value.

To properly mitigate the induced AC voltage on the pipeline to a tolerable range, as illustrated in Table 4, the optimized value of the relative permittivity of the pipeline coating increases with respect to the initial value. 


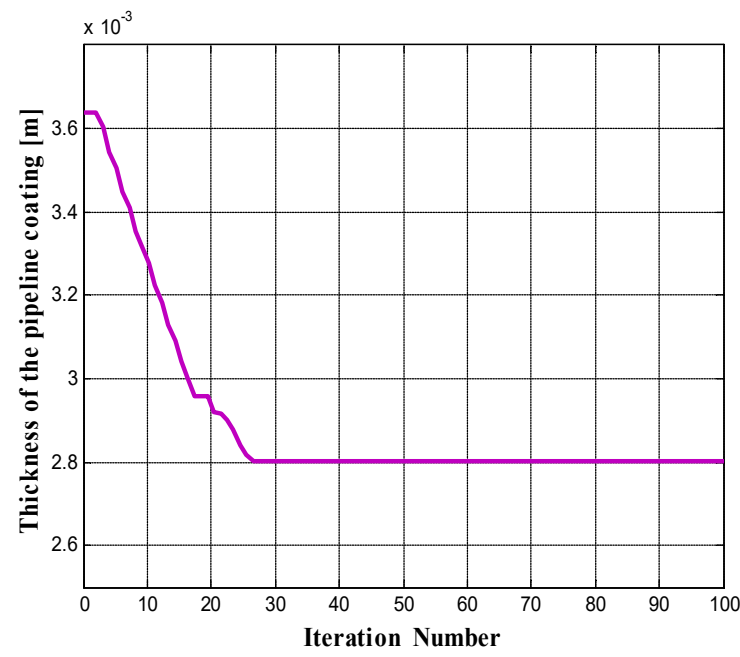

Fig. 13. Convergence to optimal value of the coating thickness of the pipeline

In order to limit the amplitude of the induced voltage on the buried metallic pipeline to a safe range, it can be seen from Table 4 that the optimized value of the coating thickness decreases compared to the initial value.

To minimize the level of the induced voltage on the buried metallic pipeline to an acceptable range, as shown in Table 4, the optimized value of the pipeline radius increases relative to the original value.

Finally, after selecting the appropriate optimization parameters, the induced voltage on the buried metallic pipeline as a function of the parallel exposure length is presented in Fig. 15. It is obvious that a very significant reduction in the induced voltage can be effected; notably at the two terminals of the buried pipeline. This induced voltage is decreased to a target value (less than $15 \mathrm{~V} \mathrm{rms}$ ). The optimization of the buried pipeline design parameters can be suggest as a proper mitigation solution to decrease the induced voltage and current on the pipeline to a safe level.

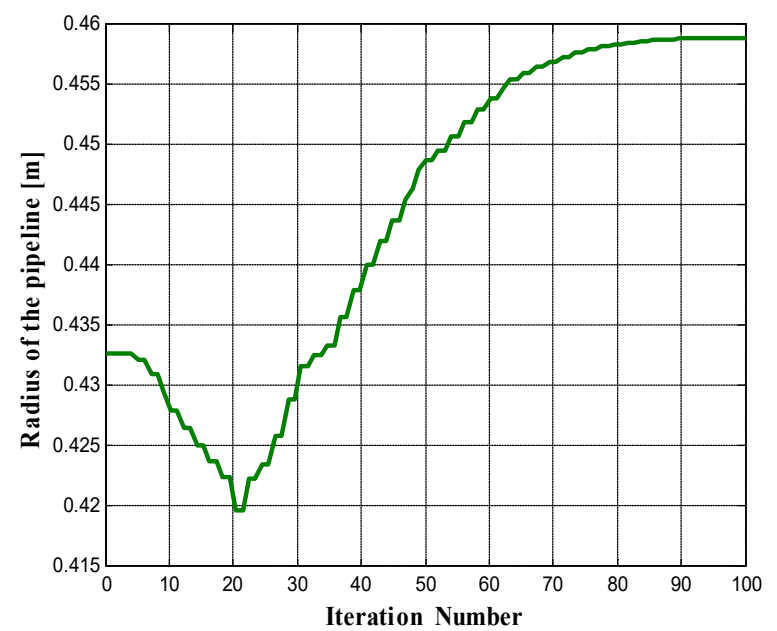

Fig. 14. Convergence to optimal value of the pipeline radius

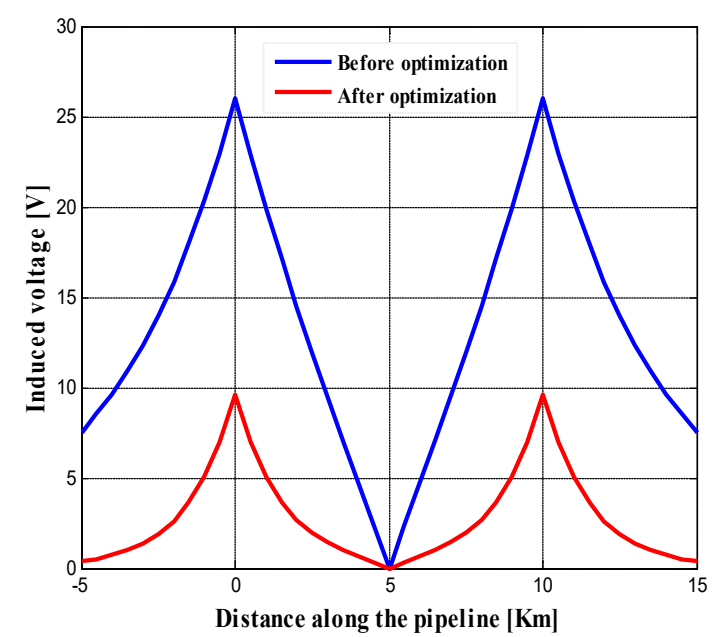

Fig. 15. Induced voltage along the buried pipeline before and after optimization

As a result, the use of an optimization procedure is very helpful for obtaining the optimal parameters of the buried pipeline for providing a mitigation system, which effectively reduces the induced voltage on the pipeline to acceptable levels to protect the operating personnel and reduce the threat of $\mathrm{AC}$ induced corrosion.

\section{CONCLUSION}

The coordination between the metallic pipeline and HV power line needs to be arranged so that the effect between them is minimized. In this paper, a methodology for computation of induced voltage on buried metallic pipeline due to the inductive coupling with nearby HV power line under steady statecondition is presented. In order to optimize the different parameters of the metallic pipeline that influence the level of this AC induced voltage, a maximization problem of an objective function is conducted using GOA, GA, DE and PSO optimization algorithms. In the first case, the obtained results have shown that the induced voltage along the length of the pipeline parallel section is higher at the two terminals of the pipeline (at the beginning and end of the pipeline) and zero at the mid-point of the pipeline length. The value of the longitudinal current is maximum on the middle point of the pipeline length and decreases at both ends of the pipeline. In the second case, the simulation results show that the GOA algorithm offers more faster convergence and better accuracy of final solution compared to other meta-heuristic algorithms. Then the statistical analysis using Friedman rank test confirm the significance and robustness of the GOA algorithm. The optimization analysis by the proposed GOA algorithm manages to choose the optimum design parameters of the metallic pipeline, which express a very good result of minimization of induced voltage on the buried pipeline that provides the personnel safety and the complete protection of the pipeline integrity. 


\section{REFERENCES}

1. Dawalibi F, Southey R. Analysis of electrical interference from power lines to gas pipelines part $\mathrm{i}$ : computational methods. IEEE Transactions on Power Delivery. 1989;14(3). https://doi.org/10.1109/61.32680.

2. Al-Badi AH, Al-Rizzo HM. Simulation of electromagnetic coupling on pipelines close to overhead transmission lines: A Parametric Study. Journal of Communications Software and Systems. 2005;1(2):116-125.

https://doi.org/10.24138/jcomss.v1i2.309

3. Micu DD, Christoforidis GC, Czumbil L. AC interference on pipelines due to double circuit power lines: A detailed study. Electric Power Systems Research. 2013;103:1-8. https://doi.org/10.1016/j.epsr.2013.04.008.

4. Chen M, Liu S, Zhu J, Xie C, Tian H, Li J. Effects and characteristics of ac interference on parallel underground pipelines caused by an $\mathrm{AC}$ electrified railway. Energies. 2018;11(9)1-24. https://doi.org/10.3390/en11092255.

5. Gouda OE, El Dein AZ, El-Gabalawy MAH. Effect of electromagnetic field of overhead transmission lines on the metallic gas pipe-lines. Electr. Power Syst. Res. 2013;103:129-136. https://doi.org/10.1016/i.epsr.2013.05.002.

6. Daconti JR. Electrical risks in transmission line pipeline shared rights of way. Power Technology. International Symposium on Environmental Concerns in Rights of Way. 2004.

7. Li Y, Dawalibi FP, Ma J. Electromagnetic interference caused by a power system network and a neighboring pipeline, Proceedings of the 62nd Annual Meeting of the American Power Conference, Chicago. 2000:311-316.

8. CIGRE Working Group 36.02. Guide on the Influence of High Voltage AC Power Systems on Metallic Pipelines. CIGRE Technical Brochure no. 095. 1995.

9. Australian New Zealand Standard, Electrical Hazards on Metallic Pipelines,Standards Australia, Standards New Zealand, (AS/NZS- 4853), (2000).

10. NACE: Mitigation of Alternating Current and Lightning Effects on Metallic Structures and Corrosion Control Systems. Report No. 21021-SG (2007).

11. EN 50443: Effects of Electromagnetic Interference on Pipelines caused by High Voltage A.C. Railway Systems and/or High Voltage A.C. Power Supply Systems. CENELEC Report No.: ICS 33.040.20; 33.100 .01 (2009).

12. Saremi S, Mirjalili S, Lewis A. Grasshopper optimisation algorithm: Theory and application. Advances in Engineering Software. 2017;105:30-47. https://doi.org/10.1016/j.advengsoft.2017.01.004.

13. Jeng-Shyang Pan. A multi-group grasshopper optimisation algorithm for application in capacitated vehicle routing problem. Data Science and Pattern Recognition, Ubiquitous International. 2020;4(1):4155.

14. El-Shorbagy MA, Ayoub AY. Integrating grasshopper optimization algorithm with local search for solving data clustering problems. International Journal of Computational Intelligence Systems. 2021;14(1): 783-793. https://doi.org/10.2991/ijcis.d.210203.008.
15. Talaat M, Hatata AY, Alsayyari Abdulaziz S, Alblawi Adel. A smart load management system based on the grasshopper optimization algorithm using the underfrequency load shedding approach. Energy Journal. 2020;190(C).

https://doi.org/10.1016/j.energy.2019.116423.

16. Steczek M, Jefimowski W, Szelag A. application of grasshopper optimization algorithmfor selective harmonics elimination in low-frequency voltage source inverter. Energies Journal. 2020;13(23):6426. https://doi.org/10.3390/en13236426.

17. Montano J, Tobón AF, Villegas JP, Durango M. Grasshopper optimization algorithm for parameter estimation of photovoltaic modules based on the single diode model, International Journal of Energy and Environmental Engineering. 2020; 11(3):367-375. https://doi.org/10.1007/s40095-020-00342-4.

18. Manpreet Kaur, Er. Ravinder Kumar. Overloading of transmission lines management by using grasshopper optimization algorithm. International Journal of Scientific \& Engineering Research. 2018;9(6):10861091.

19. Wu J, Wang H, Li N. Distributed trajectory optimization for multiple solar-powered UAVs target tracking in urban environment by adaptive grasshopper optimization algorithm. Aerospace Science and Technology. 2017;70: 497-510.

20. Jumani TA, Mustafa MW, Rasid MM, Mirjat NH, Leghari ZH, Saeed MS. Optimal voltage and frequency control of an islanded microgrid using grasshopper optimization algorithm. Energies Journal. 2018;11:3191.https://doi.org/:10.3390/en11113191.

21. Hangwei Feng, Hong Ni, Ran Zhao, Xiaoyong Zhu. An enhanced grasshopper optimization algorithm to the bin packing problem. Journal of Control Science and Engineering. 2020;2020:3894987. https://doi.org/10.1155/2020/3894987.

22. Luo Jie, Chen Huiling, Zhang Qian, Xu Yueting, Huang Hui, Zhao Xuehua. An improved grasshopper optimization algorithm with application to financial stress prediction, Applied Mathematical Modelling. 2018;64:654-668.

https://doi.org/10.1016/j.apm.2018.07.044.

23. Djekidel R, Mahi D. Calculation and analysis of inductive coupling effects for HV transmission lines on aerial pipelines. Przegląd Elektrotechniczny. 2014;90(9):151-156. https://doi.org/10.12915/pe.2014.09.39.

24. Gupta A. A study on high voltage AC power transmission line electric and magnetic field coupling with nearby metallic pipelines. Indian Institute of Science, India, 2008 (M.Sc. thesis, Dept. Elect. Eng).

25. Southey RD, Dawalibi FP. NACE International. 1998;8:564.

26. Tleis N. Power systems modelling and fault analysis theory and practice. Elsevier Ltd, 2008.

27. Hyun-Goo Lee, Tae-Hyun Ha, Yoon-Cheol Ha, JeongHyo Bae, Dae Kyeong Kim. Analysis of voltage induced by distribution lines on gas pipelines. Intematlonel Conference on Power System Technology. 2004:598-601. https://doi.org/10.1109/ICPST.2004.1460064

28. Adedeji KB. Pipeline grounding condition: A control of pipe-to-soil potential for $\mathrm{AC}$ interference induced corrosion reduction. The 25th Southern African Universities Power Engineering Conference (SAUPEC), Stellenbosch, South Africa. 2017:577-58.

29. Djekidel R, Bessedik SA, Spiteri P, Mahi P. Passive mitigation for magnetic coupling between $\mathrm{HV}$ power 
line and aerial pipeline using PSO algorithms optimization. Electric Power Systems Research. $2018 ; 165: 18-26$ https://doi.org/10.1016/j.epsr.2018.08.014

30. Leslie Bortels, Marius Purcar. Manage Pipeline integrity by predicting and mitigating high voltage AC interference. Analele Universităţii din Oradea Fascicula de Energetică. 2009; 15:2:189-195.

31. Mafarja M, Aljarah I, Faris H, Hammouri AI, AlZoubi AM. Mirjalili S. Binary grasshopper optimisation algorithm approaches for feature selection problems. Expert Systems with Applications. 2019;117:267-286. https://doi.org/10.1016/j.eswa.2018.09.015.

32. Mafarja M, Aljarah I, Heidari AA, Hammouri AI, Faris H, Al-Zoubi AM, Mirjalili A. Evolutionary population dynamics and grasshopper optimization approaches for feature selection problems. Knowledge-Based Systems. 2017;145:25-45. https://doi.org/10.1016/i.knosys.2017.12.037.

33. Ran Zhao, Hong Ni, Hangwei Feng, Xiaoyong Zhu, Yaqin Song. An improved Grasshopper optimization algorithm for task scheduling problems, International Journal of Innovative Computing, Information and Control. 2019;15(5): 1967-1987. https://doi.org/10.24507/ijicic.15.05.1967.

34. Hadeel Tariq Ibrahim, Wamidh Jalil Mazher, Osman N. Ucan, Oguz Bayat. A grasshopper optimizer approach for feature selection and optimizing SVM parameters utilizing real biomedical data sets, Neural Computing and Applications. 2019;31(2):5965-5974.

https://doi.org/10.1007/s00521-018-3414-4.

35. Hicham Deghbouch, Fatima Debbat. A hybrid bees algorithm with grasshopper optimization algorithm for optimal deployment of wireless sensor networks. Inteligencia Artificial Journal. 2021;24(67):18-35. https://doi.org/10.4114/intartif.

36. Abhishek G Neve, Ganesh M Kakandikar, Omkar Kulkarn. Application of grasshopper optimization algorithm for constrained and unconstrained test functions. International Journal of Swarm Intelligence and Evolutionary Computation. 2020;6(3):1-7. https://doi.org/10.4172/20904908.1000165 .

37. Djekidel R, Chouca A, Hadjadj A. Efficiency of some optimisation approaches with the charge simulation method for calculating the electric field under extra high voltage power lines. IET Generation, Transmission \& Distribution. 2017; 11(17):4167-4174. $\quad$ https://doi.org/10.1049/ietgtd.2016.1297.

38. Chandrasekar K, Ramana NV. Performance Comparison of GA, DE, PSO and SA Approaches in Enhancement of Total Transfer Capability using FACTS Devices. Journal of Electrical Engineering and Technology. 2012;7(4):493-500. https://doi.org/10.5370/JEET.2012.7.4.493.

39. Ibrahim Ahmed Saleh, Asmaa H, AL-Bayati, Kifaa Hadi Thanoon. Measure the software quality based on grasshopper optimization algorithm. International Journal of Computing and Digital Systems. 2020;10: 2-8.

40. Ahmed A. Ewees A, Mohamed Abd Elaziz, Essam H. Houssein. Improved grasshopper optimization algorithm using opposition-based learning, Expert Systems With Applications. 2018;112:156-172. https://doi.org/10.1016/j.eswa.2018.06.023.
41. Haoran Zhao, Huiru Zhao, Sen Guo. Short-term wind electric power forecasting using a novel multi-stage intelligent algorithm. Sustainability Journal, 10(3). 2018:881. https://doi.org/10.3390/su10030881.

42. Hastie T, Tibshirani R, Friedman J. The elements of statistical learning, data mining, inference, and prediction. Springer, New York, 2001:533. https://doi.org/10.1002/sim.1616.

43. Joaquin D, Salvador G, Daniel M, Francisco, H. A practical tutorial on the use of nonparametric statistical tests as a methodology for comparing evolutionary and swarm intelligence algorithms. Swarm and Evolutionary Computation. 2011;1(1):3-18.

44. Moayedi H, Nguyen H, Kok Foong L. Nonlinear evolutionary swarm intelligence of grasshopper optimization algorithm and gray wolf optimization for weight adjustment of neural network. Engineering with Computers. 2021;37:1265-1275.

https://doi.org/10.1007/s00366-019-00882-2.

45. Derrac J, García S, Molina D, Herrera F. A practical tutorial on the use of nonparametric statistical tests as a methodology for comparing evolutionary and swarm intelligence algorithms. Swarm and Evolutionary Computation. 2011;1(1): 3-18. https://doi.org/10.1016/j.swevo.2011.02.002.

46. Akram Seifi, Mohammad Ehteram, Vijay P. Singh, Amir Mosav. Modeling and uncertainty analysis of ground water level using six evolutionary optimization algorithms hybridized with ANFIS, SVM, and ANN. Sustainability Journal. 2020; 12(10):4023. https://doi.org/10.3390/su12104023.

Received 2021-01-03

Accepted 2021-06-09

Available online 2021-06-11

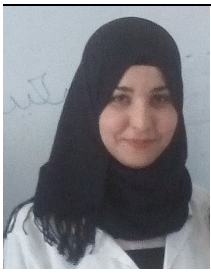

Bouallag KHADIDJA She obtained her master degree in electronics at the University of Science and Technology of Laghouat, Algeria. She is a PhD student in electromagnetic compatibility. Areas of interest: electromagnetic interference fields (EMI), high voltage engineering, and numerical modeling and simulation.

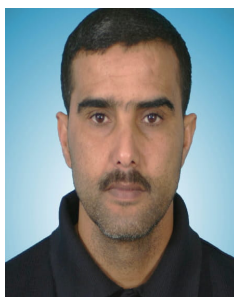

Djekidel RABAH. He graduated the University of ENSET Technique in Laghouat (Algeria), in 1991. He received the Magister and Ph.D. degrees in electrical engineering, respectively in 2010 from USTO Oran University and Laghouat University (Algeria). $\mathrm{He}$ is Professor at the University of Amar Telidji in Laghouat, Department of Electrical Engineering, Faculty of Technology, (Algeria). His research interests concern: electromagnetic interference (EMI) fields, High Voltage Engineering, and Numerical modeling and simulation

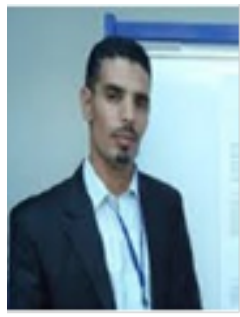

Bessidek SID AHMED. He graduated the University of ibn khaldoun in Tiaret, Faculty of (Algeria), in 2004. He received the Magister and Ph.D. degrees in electrical engineering, in 2008, respectively In 2015 from USTO Oran University (Algeria). $\mathrm{He}$ is Professor at the University of Amar Telidji in Laghouat, Department of Electrical Engineering, Faculty of Technology, (Algeria). His research interests concern: High Voltage - Insulation Materials and Dielectric - Numerical modeling and simulation, and optimization techniques of artificial intelligence-Fault diagnosis of electric machines. 